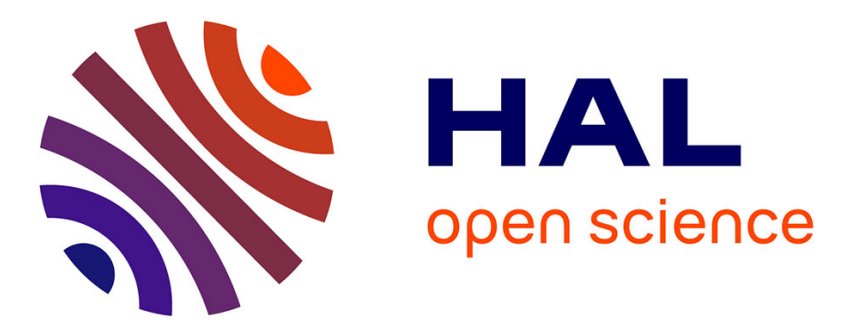

\title{
Uncertainty Management for Estimation in Dynamical Systems
}

\author{
Hana Baili
}

\section{To cite this version:}

Hana Baili. Uncertainty Management for Estimation in Dynamical Systems. Asia Pacific Conference on Circuits and Systems, Dec 2006, Singapore, Singapore. pp. 1992-1995, 10.1109/APCCAS.342278 . hal-00255900

\section{HAL Id: hal-00255900 \\ https://hal-centralesupelec.archives-ouvertes.fr/hal-00255900}

Submitted on 14 Feb 2008

HAL is a multi-disciplinary open access archive for the deposit and dissemination of scientific research documents, whether they are published or not. The documents may come from teaching and research institutions in France or abroad, or from public or private research centers.
L'archive ouverte pluridisciplinaire HAL, est destinée au dépôt et à la diffusion de documents scientifiques de niveau recherche, publiés ou non, émanant des établissements d'enseignement et de recherche français ou étrangers, des laboratoires publics ou privés. 


\title{
Uncertainty Management for Estimation in Dynamical Systems
}

\author{
Hana Baili \\ Department of Signal Processing and Electronic Systems \\ École Supérieure d'Électricité \\ 3 rue Joliot-Curie, Plateau de Moulon, Gif sur Yvette 91192, France \\ Telephone: 0033169851428, Fax:0033169851429, Email: hana.baili@supelec.fr
}

\begin{abstract}
A novel black-box model for time series of prices analysis is proposed. It is constructed using the technique of "shaping filter". The model identification is then proposed; it is based upon some stochastic calculus and a couple of results from Lévy processes theory. Neither the modeling nor the estimation parts are specific to the application, several engineering fields are concerned with this work.
\end{abstract}

\section{INTRODUCTION}

The starting point in the resolution of an estimation problem is the modeling: mathematical description of the problem. When the model comes from physics, it is said knowledge-based model, as opposed to black-box model. A model consists of a set of relations between some quantities among them appears those to be estimated. The term "dynamical" refers to the evolution in time of some quantities, and means that the model comprises at least one dynamical relation. In some model, the quantities that when fixed cause the others to be uniquely determined are called "model's data" such as the imposed conditions on the solution of an ordinary or partial differential equation, observations, controls, parameters, etc. Often some of the model's data are unknown, this is expressed by the term "uncertainty". Prior information about some unknown can be inquired. It may consist of statistics that approximate some of its moments, if it is random or of some set where it takes its values if it is deterministic. So the estimation method has to come face to face with the propagation of the information from the model's data to what is to be estimated. The management of uncertainty within dynamical models implies stochastic processes calculus; McShane's theory is used in this instance [3].

The model concerned here comes from finance, it is the following stochastic differential equation (SDE):

$$
d S_{t}=r S_{t} d t+V_{t} S_{t} d B_{t}
$$

where $S_{t}$ is the price of an asset at time $t, 0 \leq t \leq T$, and $B_{t}$ is the Brownian motion. The parameter $r$ is called interest rate, and assumed known. We observe the price at discrete equally spaced instants $t_{n}$. The stochastic process $\left(V_{t}\right)^{2}$ is called (instantaneous) volatility. It is of unknown dynamics and thus of unknown probability distribution; our objective is to estimate both of them.

The paper is organized as follows. Section 2 describes the model construction for the volatility dynamics based on prior information about such a process. Section 3 is the calibration or identification of the above model based on equation (1) and the observations. Sections 4 and 5 go further into modeling and estimation respectively. Conclusions are given in the final section.

\section{Modeling}

According to the nomenclature in the introduction, the process $\left(V_{t}\right)^{2}$ which is the quantity to be estimated, is also an unknown data in the model (1). The prior information about this unknown is its stationarity and square integrability, and a parametric model for its covariance function. Anyhow, the latter is the starting point in the estimation of the process dynamics under the form of a SDE (and thus the estimation of its probability distribution). Let the covariance function of the process $\left(V_{t}\right)^{2}$ be given by the following formula

$$
k(\tau)=D \sum_{j=1}^{m} \mathrm{e}^{-\alpha_{j}|\tau|}, \quad \alpha_{j}>0,
$$

where $D$ is the process variance. This type of covariance function allows to fit the observed time dependence in the returns; it includes short-term and long-term memory in the correlation pattern of the volatility. We consider the case where $m=2$. Then the spectral density of $\left(V_{t}\right)^{2}$ is given by the formula

$$
\begin{aligned}
s(\omega) & =\frac{1}{2 \pi} \int_{R} k(\tau) \exp (-j \omega \tau) d \tau, \\
& =\frac{1}{2 \pi} \frac{2 D\left(\alpha_{1}+\alpha_{2}\right)\left(\omega^{2}+\alpha_{1} \alpha_{2}\right)}{\left(\omega^{2}+\alpha_{1}^{2}\right)\left(\omega^{2}+\alpha_{2}^{2}\right)} .
\end{aligned}
$$

Let's denote $P(\omega)=2 D\left(\alpha_{1}+\alpha_{2}\right)\left(\omega^{2}+\alpha_{1} \alpha_{2}\right)$ and $Q(\omega)=$ $\left(\omega^{2}+\alpha_{1}^{2}\right)\left(\omega^{2}+\alpha_{2}^{2}\right)$. If we factorize the polynomial $P(\omega)$, select the factors corresponding to the roots disposed in the upper half-plane of the complex variable $\omega$ and add the factor $j^{n} \sqrt{p_{2 n}}$ ( $2 n$ and $p_{2 n}$ are the degree of $P(\omega)$ and the coefficient of $\omega^{2 n}$ in this polynomial, respectively), then we represent $P(\omega)$ in the form

$$
P(\omega)=H(j \omega) H(-j \omega), \quad \omega \in C,
$$

where

$$
\begin{gathered}
H(j \omega)=j \sqrt{2 D\left(\alpha_{1}+\alpha_{2}\right)}\left(\omega-j \sqrt{\alpha_{1} \alpha_{2}}\right), \\
=\sqrt{2 D\left(\alpha_{1}+\alpha_{2}\right)}\left(j \omega+\sqrt{\alpha_{1} \alpha_{2}}\right) .
\end{gathered}
$$


When $\omega \in R, H(-j \omega)=\overline{H(j \omega)}$ as all the coefficients of the polynomial $P(\omega)$ are real, so

$$
P(\omega)=|H(j \omega)|^{2}, \quad \omega \in R .
$$

With exactly the same reasoning applied to the polynomial $Q(\omega)$ we represent it in the form

$$
Q(\omega)=|F(j \omega)|^{2}, \quad \omega \in R,
$$

where

$$
F(j \omega)=\left(j \omega+\alpha_{1}\right)\left(j \omega+\alpha_{2}\right) .
$$

So the spectral density $s(\omega)$ is rewritten as:

$$
s(\omega)=\frac{1}{2 \pi}\left|\frac{H(j \omega)}{F(j \omega)}\right|^{2}, \quad \omega \in R .
$$

Notice now that

$$
\Phi(s)=\frac{H(s)}{F(s)}, \quad s \in C,
$$

represents the transfer function of some stationary linear system; this system is furthermore stable as all the roots of $H(s)$ and $F(s)$ are disposed in the left half-plane of the complex variable $s$. Recalling that $1 / 2 \pi$ is the spectral density of a white noise of intensity 1 , we come to the conclusion that $\left(V_{t}\right)^{2}$ may be considered as the response of the filter whose transfer function is $\Phi(s)$, to a white noise with unit intensity.

The differential equation describing such a filter is:

$\ddot{x}+\left(\alpha_{1}+\alpha_{2}\right) \dot{x}+\alpha_{1} \alpha_{2} x=\sqrt{2 D\left(\alpha_{1}+\alpha_{2}\right)}\left(\dot{u}+\sqrt{\alpha_{1} \alpha_{2}} u\right)$,

where $u(t)$ is the input and $x(t)$ is the output of the filter. If we set $X_{t}^{1}=x$ and $X_{t}^{2}=\dot{x}-\sqrt{2 D\left(\alpha_{1}+\alpha_{2}\right)} u$, we obtain the following SDE as a model for the volatility $\left(V_{t}\right)^{2}=X_{t}^{1}$ :

$$
\begin{aligned}
& d X_{t}^{1}=X_{t}^{2} d t+\sqrt{2 D\left(\alpha_{1}+\alpha_{2}\right)} d W_{t} \\
& d X_{t}^{2}=\left[-\alpha_{1} \alpha_{2} X_{t}^{1}-\left(\alpha_{1}+\alpha_{2}\right) X_{t}^{2}\right] d t- \\
& \sqrt{2 D\left(\alpha_{1}+\alpha_{2}\right)}\left[\alpha_{1}+\alpha_{2}-\sqrt{\alpha_{1} \alpha_{2}}\right] d W_{t}
\end{aligned}
$$

where $W_{t}$ is a stochastic process with independent and stationary increments (or Lévy process). It is worth noting that for $m=1$, by the same calculus, we fall in with the well documented SDE of Ornstein-Uhlenbeck for the volatility.

We assume existence for solutions of the SDE (3) when the increments of $W_{t}$ are positive (implying positivity of the volatility process $X_{t}^{1}$ ).

According to the notation of (3), (1) is rewritten in the form

$$
d S_{t}=r S_{t} d t+\sqrt{X_{t}^{1}} S_{t} d B_{t} .
$$

(4) accompanied with (3) form what we call a stochastic volatility model for the asset price. In the following we shall calibrate this model on the known data (i.e. the observations).

\section{ESTIMATION}

The lemma of Ito for the $\log$ price $Y_{t}=\log S_{t}$ together with (1) give the SDE

$$
d Y_{t}=\left(r-\frac{X_{t}^{1}}{2}\right) d t+\sqrt{X_{t}^{1}} d B_{t} .
$$

In the following we shall study integrals or aggregations of the instantaneous log-returns $d Y_{t}$, and those of the instantaneous volatility $X_{t}^{1}$, over intervals of length $\Delta$

$$
\begin{gathered}
Y_{n}=\int_{(n-1) \Delta}^{n \Delta} d Y_{t}=Y(n \Delta)-Y[(n-1) \Delta], \\
X_{n}^{1}=\int_{(n-1) \Delta}^{n \Delta} X_{t}^{1} d t .
\end{gathered}
$$

We shall prove, firstly that

$$
\operatorname{cov}\left(\left(Y_{n}\right)^{2},\left(Y_{n-k}\right)^{2}\right)=\operatorname{cov}\left(X_{n}^{1}, X_{n-k}^{1}\right),
$$

up to infinitesimals of higher order of $\Delta$, and secondly that

$$
\begin{aligned}
\operatorname{cov}\left(X_{n}^{1}, X_{n-k}^{1}\right)= \\
\frac{D}{\alpha_{1}^{2}}\left(\mathrm{e}^{-\alpha_{1}(k-1) \Delta}-2 \mathrm{e}^{-\alpha_{1} k \Delta}+\mathrm{e}^{-\alpha_{1}(k+1) \Delta}\right)+ \\
\frac{D}{\alpha_{2}^{2}}\left(\mathrm{e}^{-\alpha_{2}(k-1) \Delta}-2 \mathrm{e}^{-\alpha_{2} k \Delta}+\mathrm{e}^{-\alpha_{2}(k+1) \Delta}\right) .
\end{aligned}
$$

It follows that $D$ and $\alpha_{1}, \alpha_{2}$ may be obtained by nonlinear least squares of the difference between the approximation of the covariance function of the series of squared logreturns $\left\{\left(Y_{n}\right)^{2}\right\}_{n \geq 1}$, calculated from the observations, and the covariance function of the series of integrated volatilities $\left\{X_{n}^{1}\right\}_{n \geq 1}$, given by formula (7).

\section{A. Proof of (7)}

If we write $\xi, D$ and $k(\tau)$ respectively as the mean, the variance and the covariance function of the instantaneous volatility $X_{t}^{1}\left(=\left(V_{t}\right)^{2}\right)$, then

$$
\begin{aligned}
& \operatorname{cov}\left(X_{n}^{1}, X_{n-k}^{1}\right)=\mathrm{E}\left(X_{n}^{1} X_{n-k}^{1}\right)-\mathrm{E}\left(X_{n}^{1}\right) \mathrm{E}\left(X_{n-k}^{1}\right), \\
& =\int_{(n-1) \Delta}^{n \Delta} \int_{(n-1-k) \Delta}^{(n-k) \Delta}\left[k(u-v)+\xi^{2}\right] d u d v-(\xi \Delta)^{2}, \\
& =\int_{(n-1) \Delta}^{n \Delta} \int_{(n-1-k) \Delta}^{(n-k) \Delta} k(u-v) d u d v .
\end{aligned}
$$

If we replace $k(u-v)$ by its expression in (2), with $m=2$, we obtain the formula in (7).

\section{B. Proof of (6)}

A consequence of (5) is that

$$
Y_{n}=r \Delta-\frac{1}{2} X_{n}^{1}+\int_{(n-1) \Delta}^{n \Delta} \sqrt{X_{t}^{1}} d B_{t} .
$$

Then, up to infinitesimals of higher order of $\Delta$, it follows that

$$
\mathrm{E}\left(\left(Y_{n}\right)^{2}\right)=\xi \Delta,
$$




$$
\begin{aligned}
& \mathrm{E}\left(\left(Y_{n}\right)^{2}\left(Y_{n-k}\right)^{2}\right)=\mathrm{E}\left\{\left(\int_{(n-1) \Delta}^{n \Delta} \sqrt{X_{u}^{1}} d B_{u}\right)^{2} \times\right. \\
& \left.\left(\int_{(n-1-k) \Delta}^{(n-k) \Delta} \sqrt{X_{v}^{1}} d B_{v}\right)^{2}\right\}, \\
& =\mathrm{E}\left\{\left(\int_{(n-1) \Delta}^{n \Delta} X_{u}^{1} d u\right)\left(\int_{(n-1-k) \Delta}^{(n-k) \Delta} X_{v}^{1} d v\right)\right\}, \\
& =\operatorname{cov}\left(X_{n}^{1}, X_{n-k}^{1}\right)+(\xi \Delta)^{2} .
\end{aligned}
$$

But

$$
\begin{aligned}
& \mathrm{E}\left(\left(Y_{n}\right)^{2}\left(Y_{n-k}\right)^{2}\right)=\operatorname{cov}\left(\left(Y_{n}\right)^{2},\left(Y_{n-k}\right)^{2}\right)+ \\
& \mathrm{E}\left(\left(Y_{n}\right)^{2}\right) \mathrm{E}\left(\left(Y_{n-k}\right)^{2}\right), \\
& =\operatorname{cov}\left(\left(Y_{n}\right)^{2},\left(Y_{n-k}\right)^{2}\right)+(\xi \Delta)^{2} .
\end{aligned}
$$

Therefore (6) follows immediately.

\section{Illustration}

We observe each 10 minutes the price of some action during one month, which represents about 22 active or trading days (transactions take place from 9 a.m. to 17:30 p.m.). The variance $D$ and the rates $\alpha_{1}, \alpha_{2}$, that give a good fit between empirical approximation of the normalized covariance function and its model, amount around 556.6 and $0.02,4.91$ respectively. This confirms that a model for the volatility dynamics as an Ornstein-Uhlenbeck SDE is not satisfactory, and points towards the use of sum of exponentials with different persistence rates when modeling the covariance function of the volatility.

\section{Further into MOdELING}

In this section we shall develop the link between the stationary solution of an SDE of the same type as (3) and the generating or driving process in this SDE (i.e. the Lévy process). We need in this instance to introduce the notion of self-decomposability of a probability distribution; more precisely, we need to know the relation between this property and Lévy processes.

\section{A. Theorem}

A probability distribution is self-decomposable, i.e. its characteristic function $\phi$ satisfies

$$
\phi(\lambda)=\phi(\rho \lambda) \phi_{\rho}(\lambda)
$$

for some family of characteristic functions $\phi_{\rho}, \rho \in(0,1)$, if and only if there is a Lévy process $W_{t}$ such that the random variable

$$
\int_{0}^{\infty} \exp (-A s) B d W_{s}
$$

follows this distribution, for some $A$ and $B$.

\section{B. Proposition}

Let $W_{t}$ be a Lévy process, denote the Lévy measure of $W(1)$ by $P$, suppose moreover that $P$ has $p$ as density. The specification

$$
X_{t}=\int_{-t}^{\infty} \exp [-A(s+t)] B d W_{s}
$$

determines a stationary process if and only if $p$ satisfies

$$
\int_{|x| \geq 1} \log |x| p(x) d x<\infty .
$$

It follows, by change of the integration variable, and from increments stationarity of a Lévy process that

$$
\int_{0}^{\infty} \exp (-A s) B d W_{s},
$$

has the same probability distribution as $X(t)$ for all $t>0$. So according to the theorem, this probability distribution is self-decomposable. It is worth noting that the process $X_{t}$ of the proposition is a solution of the following SDE:

$$
d X_{t}=-A X_{t} d t+B d W_{t}
$$

and that SDE (3) is of this form with

$$
\begin{aligned}
& A=\left(\begin{array}{ll}
0 & -1 \\
\alpha_{1} \alpha_{2} & \alpha_{1}+\alpha_{2}
\end{array}\right), \\
& B=\sqrt{2 D\left(\alpha_{1}+\alpha_{2}\right)}\left(\begin{array}{l}
1 \\
\alpha_{1}+\alpha_{2}-\sqrt{\alpha_{1} \alpha_{2}}
\end{array}\right) .
\end{aligned}
$$

The results in subsections $A$ and $B$ are promoted by [1][2], where one-dimensional case is treated. We admit the results for multidimensional case (the matrices $A$ and $B$ are $n \times n$ and $n \times 1$ respectively).

\section{FURTHER INTO ESTIMATION}

In the present section we shall achieve our objective about estimating both the dynamics and the probability distribution of the volatility modeled as a stochastic process. We proceed in this way: we specify the Lévy measure of the SDE generating process, we ensure that the condition of the proposition applies, i.e. the SDE has a stationary solution and then we recover the probability distribution of the latter. Our approach to do that is to simulate the stochastic process $X_{t}$. It is easy to show that

$$
\begin{aligned}
& X(t)=\exp (-A t) X(0)+ \\
& \left(\int_{-t}^{0} \exp (-A s) d W_{s}\right) \exp (-A t) B, \\
& =\exp (-A t) X(0)+\left(\int_{0}^{t} \exp (A s) d W_{s}\right) \exp (-A t) B .
\end{aligned}
$$

So we have to simulate from

$$
\int_{0}^{t} \exp (A s) d W_{s} .
$$


We shall use infinite series representation of this type of integrals [1][2]. The desired result is that: for an arbitrary function $f$, when the integral exists,

$$
\int_{0}^{t} f(s) d W_{s}=\sum_{i=1}^{\infty} f\left(u_{i} t\right) P^{-1}\left(\frac{t_{i}}{t}\right),
$$

where $\left\{u_{i}\right\}$ is an i.i.d. sequence of random variables following the uniform distribution on $[0,1]$, and $\left\{t_{i}\right\}$ are arrival times of a Poisson process with intensity $1 . P^{-1}$ denote the inverse of the upper tail integral function

$$
P^{+}(x)=P([x, \infty)) .
$$

\section{A. Illustration}

Let the Lévy measure $P$ of $W(1)$ have support $R_{+}$and be such that

$$
P^{+}(x)=\nu \exp (-\alpha x),
$$

with $\alpha>0$ and $\nu>0$. Then

$$
p(x)=\alpha \nu \exp (-\alpha x),
$$

which satisfies (8). In this case $P^{+}$can be analytically inverted:

$$
P^{-1}(x)=\max \left\{0,-\frac{1}{\alpha} \log \left(\frac{x}{\nu}\right)\right\} .
$$

Consider the histogram approximating the probability distribution of the squared log-return on 1 unit-of-time; it is also an approximation for the probability distribution of the volatility process $X_{t}^{1}$. It is easy to see that by elementary handling of equation 5. So this histogram is our reference for identifying $\alpha$ and $\nu$; we find $\alpha=1$ and $\nu=887.8810^{-5}$.

\section{CONCLUSION}

A probabilistic approach to manage uncertainty for estimation in dynamical models is proposed when illustrated on an application from financial engineering: volatility estimation. We consider the volatility as a stochastic process; we construct a model for its dynamics under the form of a stochastic differential equation, driven by a Lévy process, and obtain at one go the probability distribution of the volatility process. The conformity between theoretical and practical issues, together with the accuracy of results within low simulation cost, prove to my satisfaction the performance of the approach.

\section{REFERENCES}

[1] O. E. Barndorff-Nielsen and N. Shephard, Non-Gaussian OrnsteinUhlenbeck-based models and some of their uses in financial economics, Journal of the Royal Statistical Society, Series B (2001), 63, pp. 167241.

[2] O. E. Barndorff-Nielsen and N. Shephard, Econometric analysis of realized volatility and its use in estimating stochastic volatility models, Journal of the Royal Statistical Society, Series B (2002), 64, 253-280.

[3] E. McShane, Stochastic Calculus and Stochastic Models, Academic Press, 1974

[4] V. S. Pugachev, I. N. Sinitsyn, Stochastic Systems, Theory and Applications, JOHN WILEY \& SONS, 1987. 